\title{
DIE ERSTELLUNG EINES BLOGS ÜBER INDONESISCHE SPEZIALITÄTEN
}

\author{
Sri Yulianti \\ Siti Kudriyah \\ Tanti Kurnia Sari
}

\begin{abstract}
AUSZUG
Das Ziel dieser Untersuchung ist ein Blog über Indonesische Spezialitäten zu erstellen. In dieser Untersuchung wird die deskriptive qualitative Methode angewendet. Diese Untersuchung wird in der Bibliothek der Fakultät für Sprache und Kunst der Staatlichen Universität von Medan durchgeführt. Der Prozess der Erstellung eines Blogs über Indonesische Spezialitäten besteht aus der Erklärung der Phasen von Theorie Plomp. Darunter sind: (1) die Beobachtungsphase, (2) die Designphase, (3) die Realisierungsphase, und (4) die Test-, Evaluations-und Revisionsphase. Die Indonesische Spezialitäten will in einem Blog auf Deutsch vorgestellt werden. Der Name des Blogs ist www.sriyuliantimhs.blogspot.co.id. Es gibt ca 10 Posting, die in einem Blog publiziert werden, sind: Nani Arsik, Rendang, Gudeg, Ayam Betutu, Papeda, Opor Ayam, Karedok, Pempek, Soto Betawi und Lempah Kuning. In jeder Posting besteht aus die Texte und Bilder. Der Text enthält die Erklärung die Indonesische Spezialitäten. Die Erstellung des Blogs wird den Experten Evaluation gemacht. Die Ergebnisse der Evaluation zeigt, dass das Blog gut ist. Der Inhalt und Thema des Blogs sind interessant zum Lesen. Die Darstellung ist deutlich. Die Design des Blogs ist interessant mit der vorhandene Unterteilung des Menüs. Die Farbe und der Hintergrund des Blogs kann den Lesern die Lust zum Lesen wecken.
\end{abstract}

Schlüsselwörter: Erstellung des Blogs, die Indonesische Spezialitäten EINLEITUNG

Blog ist die Abkürzung von Weblog. Blog wurde zuerst von Pyra Labs am 23. August 1999 bekannt gemacht. Rachmanto (vgl. 2015: 3) stellt fest, dass der Blog eine Web-Anwendung ist, die die Form von Schriften (veröffentlicht als Post) auf einer öffentlichen Webseite ähnelt. Ein Blog gibt eine gute Möglichkeit, sich Zeit zu nehmen und dabei mit weit entfernten Freunden in Kontakt bleibt. Das Blog kann Links, Bilder, Videos und Audios enthalten. In einem Blog gibt es nicht nur Informationen zur Erfahrungsberichte sondern auch Kurzgeschichten, Tipps, Gesundheit, Bildung zum Tourismus und Sozialarbeit. Viele Menschen lesen das Blog als Referenzmaterial für verschiedene Themen. Ein Blog kann verschiedene Funktionen erfüllen, z.B. Kommunikationsmittel oder Werbeträger.

Jeder kann ein Blog machen. Man braucht keine technische Fähigkeit oder grundlegende Fähigkeit des Journalismus, um die Informationen im Blog zu veröffentlichen. Einige Blogs konzentrieren sich auf ein bestimmtes Thema. Das 
Bloggen ist nicht nur ein guter Weg, um gute Schriftsteller zu werden, sondern auch der schnellste Weg, ein Schriftsteller in den Massenmedien zu werden. In dieser Untersuchung will ein Blog über Indonesische Spezialitäten gemacht werden. Dieses Blog will sich die Indonesische Spezialitäten vorstellen, die in Indonesien populär sind. Z.B Indonesisches Essen: Nani Arsik (Nord Sumatra), Rendang (West Sumatra), Gudeg (Yogyakarta), Ayam Betutu (Bali), Papeda (Papua). Indonesische Spezialitäten können durch das Blog in der ganzen Welt gekannt werden. Die leser können die Geschichte der Küchen wissen und auch probieren selbs zu kochen, denn in dem Blog gibt es auch das Rezept der Küchen.

In Indonesien gibt es viele Orte an denen Deutsch gelernt wird, zum Beispiel in den Schulen, in Sprachkursen, und in den Universitäten. Die Studenten, die Deutsch lernen, lernen natürlich nicht nur die Grammatik, Vokabeln und die vier Sprachkompetenzen (Lesen, Hören, Sprechen und Schreiben), sondern sie lernen auch etwas über Sehenwürdigkeiten und Kulturen.

Indonesien hat auch verschiedenen kulinarischen Spezialitäten, die aus verschiedenen Stämmen Kommen. Indonesische Spezialitäten ist eine der Elemente der Kultur, die in verschiedenen Regionen in Indonesien verstreut sind. Jede Regionale hat verschiedene Essens mit Variation, Funktion und Art der Präsentation. Indonesien ist nicht nur für seine Kultur, sondern auch für seine Küche berühmt. In Indonesien gibt es viele köstliche Spezialitäten aus den einzelnen Regionen jeweils. In einer Region spiegelt in der Regel den Charakter der Menschen.

Basierend auf den oben genannten Problemen ist es interessant und sinnvoll einen Blog über indonesische Spezialitäten zu erstellen.

\section{THEORETISCHE GRUNDLAGE}

\section{Der Begriff der Erstellung}

Hutagalung (vgl. 2016) stellt fest, dass die Erstellung eine Forschung ist, deren Ziel die Erstellung eines Produktes ist. Eine Erstellung ist ein Prozess oder eine Methode, etwas besser oder perfekt zu entwickeln. Sugiono (2012) sagt, dass die Erstellung eine Forschungsmethode ist, die benutzt wird, um ein bestimmtes Produkt herzustellen und die Wirksamkeit dieser Produkte zu testen. Eine auf 
Studia: Journal des Deutschsprogramms

p-ISSN 2301-6108

e-ISSN 2654-9573

https://jurnal.unimed.ac.id/2012/index.php/studia/index

Bedarfanalyse basierte Untersuchung, wird verwendet, ein bestimmtes Produkt herzustellen und um die Wirksamkeit dieses Produkts zu testen.

\section{Der Begriff des Blogs}

Der Blog ist die Abkürzung von Web und log. Bloggen ist die schriftstellerische Tätigkeit mithilfe des Medium Blog oder eines Website. Blogger sind die Menschen, die diese Tähigkeit durchführen. Quible (Vgl. 2005) definiert den Blog als eine Abkürzung für Weblog, worunter man auch die Log- Dateien auf dem erscheinenden Blog verstehen kann, wenn man Internet-Seiten besucht. Suryana und Matamaya (vgl. 2008) behaupten, dass der Blog eine 
Webapplikation ist, die aus Texten als Post genannt besteht. Diese Applikation ist kostenlos. Posten können Informationen, Fotos, Geschichten, Bilder und auch Video sein. Supriyanto (vgl. 2009) stellt fest, dass der Blog wie ein Tagebuch ist, der über Erfahrungen, Ideen oder auch Geschichten des Blogschreibers berichtet. Man kann sich sehr einfach für einen Blog registrieren. Deshalb haben heute viele Menschen einen eigenen Blog zu bestimmten Themen. Die Themen des Blogs sind dabei unterschiedlich, z..B: zu Ausbildung, Gesundheit, Sport, Mode und Tourismus, Sodass man ziemlich einfach verschiedene Informationen über sie erhalten kann.

\section{Indonesische Spezialitäten}

Kulinarischer Tourismus macht immer Spa $\beta$. Wir können verschiedene lokalen Lebensmittel finden, die es nicht notwendigerweise an einem anderen Platz gibt. Ebenso sind die Nahrungsmittelzubereitungsmethoden mit der umgebenen Kultur verbunden. Einzigartig aber voller Geschmack. Wenn man auf den Inseln in Indonesien reist, kann man viele leckere Gerichte finden. In Sumatra werden zum Beispiel Gerichte serviert, die mit vielen Gewürzen zubereitet werden. Auf Java und Bali werden einfachere Gerichte, serviert, aber auch sie haben ihren eigenen regionalen Charakter. Das ist auch so in Kalimantan, Sulawesi und Ostindonesien. Diese Gerichte sind auch sehr interessant.

Natürlich geben die Vielfalt der Stämme, der ethnischen Zugehörigkeit, der Sprache und Kultur Indonesien eine große Auswahl an schmackhaften kulinarischen Spezialitäten. Als Beispiele seien hier: Nani Arsik aus Nord Sumatra, Rendang aus West Sumatra, Yogyakarta mit seinem unverwechselbaren Gudeg, Bali mit Ayam Betutu, Papeda von Papua, Pempek aus Süd-Sumatra, Opor Ayam aus Zentral-Java, Karedok aus West-Java, Soto Betawi aus Jakarta und Lempah Kuning aus Bangka genannt.

\section{Das Entwicklungsmodell}

Um ein Blog über Indonesische Spezialitäten zu erstellen, interessiert sich das Entwicklungsmodell von Plomp, da es leichter als die Entwicklungsmodell von 
anderen Experten ist. Plomp (in Hutagalung) hat das Entwicklungsmodell in vier Stufen, nämlich (1) Die Beobachtungsphase, (2) die Designphase, (3) die Realisierungsphase (4) die Test-, Evaluation-, Revisionsphase.

\section{UNTERSUCHUNGSMETODE}

Diese Untersuchung ist eine Erstellungsuntersuchung, die der Webseite zuerstellten. Diese Untersuchung benutzt die deskriptive qualitative Methode.

\section{Die Daten und die Datenquelle}

Die Daten in dieser Untersuchung sind einige ausgewählte indonesische, wie Nani Arsik, Rendang, Gudeg, Ayam Betutu und Papeda. Die Daten stammen von einem Blog über indonesische Spezialitäten.

\section{Die Datenbeschreibung}

In dieser Untersuchung wird Plomps Theorie benutzt. In Kapitel II wurde diese Theorie bereits erklärt. Im Folgenden ist eine Skizze von Plomps Theorie dargestellt. Diese Theorie wird in dieser Untersuchung angewendet.

\section{ERGEBNIS DER UNTERSUCHUNG Der Prozess der Erstellung}

\section{Die Beobachtungsphase}

Dies ist die erste Phase, um den Blog über Indonesische Spezialitäten zu erstellen. In dieser Phase wird eine Recherche gemacht, um herauszufinden, ob es bereits einen Blog über Indonesische Spezialitäten gibt. Basierend auf der Recherche wird festgestellt, dass es bisher nur wenige Blogs über Indonesische Spezialitäten gibt. Die meisten Blog befassen sich mit einer kurzen Erklärung über Indonesische Spezialitäten, zum Beispiel mit Geschichte oder Erfahrungen von einigen Deutschen, die Indonesische Spezialitäten kochen.

Mit meinem Blog möchte ich weiter gehen, als die bisher vorhandenen Blogs. Das heißt, dass nicht nur die Geschichte und die Kochenerfahrungen erklärt werden, sondern auch weitergehende Informationen über Indonesische Spezialitäten sowie deren Rezepte. Dazu werden die Informationen aus Büchern, von Youtube und über Google gesammelt. Die gesammelten Daten werden auf Indonesisch beschrieben. 
Studia: Journal des Deutschsprogramms

p-ISSN 2301-6108

e-ISSN 2654-9573

https://jurnal.unimed.ac.id/2012/index.php/studia/index

Die Zehn Blog vorgestellten indonesische Spezialitäten sind:

a) Na Niarsik (Nord-Sumatra)

Nord-Sumatra ist für „Na Niarsik” bekannt. Es ist ein indonesisches Fischgericht von den Toba-Batak in Nord-Sumatra. Meistens werden Goldfisch und Gewürze benutzt, das Gericht hat einen würzigen Geschmack. Es schmeckt sauer, bitter und scharf zugleich.

b) Rendang (West-Sumatra)

Rendang ist ein typisches Gericht aus West Sumatra und es ist weltweit berühmt. Rendang besteht aus Fleisch, das mit Kokosmilch gekocht wird. Rendang wird meistens bei Zeremonien serviert, um die Gäste zu ehren. Außerdem wird Rendang auch bei festlichen Anlässen wie Hochzeiten, Zeremonien und Festen (Eid al-Fitr) zubereitet.

c) Gudeg (Yogyakarta)

Yogyakarta ist auch als Gudeg Stadt bekannt, denn diese Stadt hat ein typisches Gericht, das Gudeg heißt. Das Gericht besteht aus jungen Jackfrüchten, die mit Kokosmilch gekocht werden und es hat ein besonderes Aroma. Gudeg wird meistens mit Reis serviert, aber man kann es auch mit Brei essen. Gudeg wird meistens mit Kokosmilch, Hühnerfleisch, Eiern, Tempe, Tofu und Sambal serviert.

d) Ayam Betutu (Bali)

Bali hat nicht nur schöne Strände, sondern auch eine sehr schmackhafte und exotische Mahlzeit, die Betutu heisst. Betutu ist ein Gericht mit Huhn oder Ente, das gewürzt und in der Glut von Reishülsen gebacken wird. Dieses Gericht ist auch reich an Gewürzen. Die Gewürze werden dann mit gekochten und pürierten Maniokblättern gemischt und dann in die Bauchhöhle des Huhns oder der Ente gefüllt. Manchmal wird „Betutu” auch in Bananenblätter gewickelt und dann wird es gegrillt.

e) Papeda (Papua)

Ein typisches Gericht und Grundnahrungsmittel in Papua ist Papeda. Papeda wird jeden Tag als Hauptmahlzeit gegessen. Papeda ist ein traditionelles Gericht, 
das aus Sago gemacht wird.

f) Pempek (Süd-Sumatra)

Verarbeitete Fische wurde einer der beliebtesten Lebensmittel aus Indonesien. Pempek ist eine der typischen Speisen aus Süd-Sumatra, vor allem in Palembang. Pempek ist aus Sagomehl und Fisch, Pempek wird mit schwarzem Essig Sauce-braun serviert, die süß und sauer schmeckt,.

g) Opor Ayam (Zentral-Java)

Opor Ayam ist ein sehr bekanntes Gericht in Indonesien. Opor Ayam stammt, aus Java, vor allem aus Zentral- Java und Ostjava. Trotzdem ist dieses Gericht auch in anderen Orten weit verbreitet. Opor Ayam ist eigentlich ein gekochtes Huhn, dem dicke Kokosmilch und verschiedene Gewürze wie Zitronengras, Galgant und andere beigegeben werden.

h) Karedok (West-Java)

Karedok ist ein typisches Gericht aus Java, genauer gesagt aus West-Java. Diese Gericht ist ungewöhnlich für die indonesische Küche, denn das Gemüse bleibt roh und wird nicht gekocht, so dass der Nährstoffgehalt und die Faser erhalten bleiben. Das rohe Gemüse wie güne Bohnen, Kohl, runde Auberginen, Sprossen, Gurken und Basilikumblätter wird dann mit einer leckeren und leicht würzigen Erdnuss-Sauce übergossen und bieten eine außergewöhnliche Sensation beim Essen.

\section{i) Soto Betawi (Jakarta)}

Soto Betawi ist eine beliebte Suppe in Jakarta. So wie Soto Madura und Soto Sulung verwendet man auch für Soto Betawi Innereien. Außer Innereien werden oft auch andere Organe benutzt, wie Augen und auch die Leber.

\section{j) Lempah Kuning (Bangka)}

Lempah kuning ist ein Rezept, das ursprünglich aus Bangka Belitung stammt und bei, den Menschen in Bangka Belitung sehr beliebt ist. Die Küche hat einzigartiges Geschmack und ist auch sehr lecker, besonders wenn es mit Reis 
serviert wird. Lempah kuning wird aus dem Kerapufisch mit vielfältigen Gewürze zubereitet.

\section{Die Designphase}

In der zweiten Phase werden die Ergebnisse der ersten Phase genutzt und verarbeitet. In dem Übersetzungsprozess werden relevante Wörter ausgewählt, um die Indonesischen Spezialitäten $\mathrm{zu}$ beschreiben. Nachdem die Informationen übersetzt wurden, werden sie danach in einem Blog mit umfassenden Erklärungen und Fotos dargestellt.

In dieser Phase wird das Konzept des Blogs entworfen, um das interessanten und ansprechenden Blog zu erstellen. Das Konzept des Blogs wird in dem vorhandenen Blog realisiert, nämlich www.sriyuliantimhs.blogspot.co.id. Es ist das Blogspot, das schon registriert worden.

\section{Realisierungsphase}

Konzepte, die in der zweiten Phase geplant wurden, werden in dieser Phase realisiert. Die Bilder werden den passenden Themen zugeordnet und die Texte werden übersetzt.

1. Die Ergebnisse des Designs des Blogs

Das Design des Blogs ist vielfältig und kann entworfen werden. Das Design des Blogs besteht aus Menü, dem Theme, den Bildern, dem Hintergrund, usw. Sie können entwickelt werden, um den Blog ansprechend zu gestalten.

2. Die Ergebnisse der entworfenen Menüs

In diesem Teil wird das Menü erstellt. Das Menü besteht aus Home, Indonesische Spezialitäten, Galerie und Profil. Sie können auf die nächste Seite klicken, um ein neues Thema zu öffnen.

\section{a. Home}

Wenn Leser auf Home klicken, bekommen sie einführendedie Informationen über den Blog und Indonesische Spezialitäten.

\section{b. Indonesische Spezialitäten}

Wenn auf das Menü "Indonesische Spezialitäten" geklickt wird, öffnen sich 
zehn Gerichte zur Auswahl: Nani Arsik, Rendang, Gudeg, Ayam Betutu, Papeda, Pempek, Opor Ayam, Karedok, Soto Betawi und Lempah Kuning.

\section{c. Galerie}

Wenn auf die Galerie geklickt wird, dann kann man die Bildern mit den Erklärungen sehen.

d. Profil

Im unteren Teil des Hauptmenus befindet sich das Profil der Bloggerin.

3. Ergebnisse der erstellten Blogpostings (Artikel)

Nachdem das Design des Blogs realisiert worden ist, werden die Daten im einem Blog eingestellt. Bevor die Daten publiziert werden, können sie nochbearbeitet werden. Es wird gemacht um die gute Artikel oder Posting zu erhalten. Zehn Postings wurden bereits, publiziert.

\section{Die Test-, Evaluation-, und Revisionsphase}

Dies ist der letzte Schritt bei der Erstellung eines guten Blogs. Der bereits erstellte Blog wird geprüft, da man herausfinden möchte, ob der Blog bereits gut genug ist oder noch verbessert werden sollte. Meist wird der Blog dafür von Experten geprüft. Diese Experten sind deutsche Muttersprachler und Blogsdesigner, die sich mit Blogdesign auskennen. Nach der Evaluierung durch die Experten wird der Blog verbessert. Anschließend wird der Blog öffentlich zugänglich gemacht, sodass auch Leser Kritiken oder Kommentare äußern können. Eine Muttersprachlerin hat die Daten korrigiert, und Verbesserungsvorschläge abgegeben, die Muttersprachlerin behauptet, dass das Blog sehr interessant ist.

\section{SCHLUSSFOLGERUNG}

Nach den Untersuchungsergebnissen fallen die Schlussfolgerungen folgendermaßen aus:

1. Der Prozess der Erstellung eines Blogs über Indonesische Spezialitäten. Dieser Prozess besteht aus der Erklärung der vier Phasen nach Plomp: (1) die Beobachtungsphase, (2) die Designsphase (3) die Realisierungsphase und (4) die Test-, Evaluations-und Revisionsphase.

Der Blog wurde mit Blogspot.com erstellt, die Blog- Adresse ist www.sriyuliantimhs.blogspot.co.id. 
2 Das Ergebnis der Erstellung eines Blogs über Indonesische Spezialitäten. Der Blog über indonesische Spezialitäten besteht aus zehn Postings, die die Darstellung und Beschreibung indonesischer Spezialitäten enhält. Die Beschreibung ist auf Deutsch verfasst. Die Indonessichen Spezialitäten, die im Blog publiziert wurden, sind: Nani Arsik, Rendang, Gudeg, Ayam Betutu Papeda, Pempek, Opor Ayam, Karedok, Soto Betawi und Lempah Kuning. Die Experten, die auf diesen Blog eine Evaluation machen, sind die deutsche Muttersprachler und Informatikes. Anschließend wird der Blog öffenlich zugänglich gemacht, sodass auch Leser Kritiken oder Kommentare äußern können. Basierend auf der Meinungen von Experten können geschlossen werden, dass das Blog über indonesische Spezialitäten gut ist. Es hat auch interessante Inhalt und Thema und die Darstellung ist deutlich mit den Bildern.

\section{LITERATURVERZEICHNIS}

Enterprise, Jubilee. 2009. Google untuk Guru. Jakarta: PT Elex Media Komputindo Kelompok Gramedia, Anggota IKAPI.

GmbH, Kiepert. 2014. Bloggst du schon?!. Stuttgart: frechverlag.

http://www.scil.unisg.ch/ /media/internet/content/dateien/instituteundcenters/iwpscil/arbeitsberichte/scilab-12.pdf\#page=71, gelessen am 27 Februar 2017, um $12.00 \mathrm{Uhr}$.

http://staff.uny.ac.id/sites/default/files/PENELITIAN\%20PENGEMBANGAN.pdf, gelessen am 1 März 2017, um 14.00 Uhr.

https://www.academia.edu/10224224/BLOG_SEBAGAI_MEDIA_PEMBELAJAR AN_DAN_KREATIVITAS, gelessen am 5 März 2017, um 09.00 Uhr. 
Studia: Journal des Deutschsprogramms

p-ISSN 2301-6108

e-ISSN 2654-9573

https://jurnal.unimed.ac.id/2012/index.php/studia/index

Khadafi, Rizal. 2008. Atlas Kuliner Nusantara Makanan Spektakuler 33 Provinsi. Jakarta: Bukune.

Oya, Suryana, Matama Studio.2008. Membangun Blog Wordpress. Jakarta : Elex Media Komputindo Kelompok Gramedia, Anggota IKAPI.

Plomp, T. and Vieven, N. (ed). 2007. An Introduction to educational Design Research. Enschede: SLO

Rachmanto, Ricky. 2015. Blogger untuk Pemula. Jakarta: PT Elex Media Komputindo Kelompok Gramedia, Anggota IKAPI.

Sugiono, Dr, Prof, Bud E. 2012. Metode Penelitian Pendidikan Pendekatan Kuantitatif, Kualitatif, dan R\&D. Bandung: ALFABETA, cv.

Supriyanto, Ahmad. 2013. Membuat Blog dengan Blogspot Lanjutan. Jakarta : Elex Media Komputindo Kelompok Gramedia, Anggota IKAPI.

Willsen, Lea. 2015. Blogger Blogspot untuk Pemula Kreatif. Jakarta : Elex Media Komputindo Kelompok Gramedia, Anggota IKAPI. 